\title{
Lesión cerebral hipóxico-isquémica secundaria a parada cardiaca en un lesionado medular agudo
}

\author{
Hypoxic-ischemic brain injury secondary \\ to cardiac arrest in acute spinal cord injury
}

\begin{abstract}
Francisco Ramón Pampín-Huerta'1, Beatriz Muíño-Vidal², Antonio Montoto-Marqués³, Sebastián Salvador-de la Barrera ${ }^{3}$.

${ }^{\top}$ Médico especialista en Medicina Intensiva. Unidad de Reanimación y Cuidados Intensivos. Hospital HM Modelo. A Coruña. España. ${ }^{2}$ Médico especialista en Medicina Física y Rehabilitación. Servicio de Rehabilitación. Hospital Universitario Lucus Augusti. Lugo. España. ${ }^{3}$ Médico especialista en Medicina Física y Rehabilitación. Unidad de Lesionados Medulares. Complejo Hospitalario Universitario de A Coruña. A Coruña. España.
\end{abstract}

\section{Resumen}

La lesión medular es un factor de riesgo importante para la enfermedad tromboembólica venosa, entidad que engloba la trombosis venosa profunda y el tromboembolismo pulmonar, siendo este una causa potencialmente reversible de parada cardíaca.

Presentamos el caso de un varón joven con lesión medular completa (grado ASIA A) con nivel C6 que sufre una parada cardíaca por tromboembolismo pulmonar masivo secundario a trombosis venosa profunda en extremidad inferior izquierda en la $6^{a}$ semana de ingreso. Exponemos la incidencia de ambas entidades en la lesión medular aguda así como su fisiopatología, profilaxis y tratamiento; destacamos la importancia de la identificación de la parada cardíaca e inicio precoz de las maniobras de reanimación cardiopulmonar por el personal sanitario y analizamos las consecuencias de la lesión cerebral hipóxico-isquémica derivada de una parada cardíaca recuperada.

Palabras clave: Lesión medular. Trombosis venosa profunda. Tromboembolismo pulmonar. Parada cardíaca.

\section{Caso clínico}

La parada cardíaca (PC) es una de las situaciones más temidas para los profesionales sanitarios; puede presentarse en cualquier paciente hospitalizado, por sus comorbilidades previas o por la patología que condiciona su ingreso.

La lesión de la médula espinal es un factor de riesgo importante para la enfermedad tromboembólica venosa (ETEV): engloba la trombosis venosa profunda (TVP) y el tromboembolismo pulmonar (TEP). ${ }^{1}$ La literatura pone de manifiesto una incidencia muy variable de TVP en la lesión medular aguda (LMA), ${ }^{2-5}$ alcanzando hasta un $7 \%$ con profilaxis. ${ }^{3}$ En esta población la mitad de los pacientes con TVP pueden presentar un TEP. ${ }^{2}$ La incidencia del TEP en la LMA oscila entre el 5-12\%, 4,5 es una de las causas potencialmente reversibles de PC que se puede presentar en una Unidad de Lesionados Medulares (ULM) y la lesión cerebral hipóxico-isquémica (LCHI) una de las posibles consecuencias tras la recuperación de la circulación espontánea (RCE). ${ }^{6}$

Varón de 22 años sin antecedentes que sufre traumatismo vértebro-medular por vuelco del automóvil. Los Servicios de Emergencias evidenciaron incapacidad para movilizar las cuatro extremidades, iniciaron pauta de metilprednisolona (NASCIS III) ${ }^{7}$ y lo trasladaron al hospital, donde se diagnosticó fractura-luxación C6-C7 con lesión medular (figura1A), y desde ahí al centro de referencia con ULM. Ingresó con una exploración neurológica correspondiente a lesión medular completa (grado ASIA A) con nivel sensitivo C4 y motor C6. Se completó la pauta de metilpredniso-

\begin{abstract}
Spinal cord injury is a strong risk factor for venous thromboembolism, entity that encludes deep vein thrombosis and pulmonary embolism, this being a potentially reversible cause of cardiac arrest. We report the case of a young male with complete spinal cord injury (ASIA grade A) level C6 suffering cardiac arrest by massive pulmonary embolism secondary to deep vein thrombosis in the left lower limb in the 6th week of admission. We report the incidence of both entities in acute spinal cord injury and their pathophysiology, prevention and treatment; We emphasize the importance of identifying cardiac arrest and early initiation of cardiopulmonary resuscitation by health workers and analyze the consequences of hypoxic-ischemic brain injury resulting from cardiac arrest recovered. Keywords: Spinal cord injury. Deep vein thrombosis. Pulmonary thromboembolism. Cardiac arrest.
\end{abstract}

Iona y se colocó compás de tracción craneal para reducir la fractura-luxación. Se inició profilaxis de TVP a las 48 horas con 3500 UI de bemiparina; se realizó de modo programado una artrodesis vertebral con osteosíntesis C6-C7 (figura1B) el $18^{\circ}$ día y se inició la sedestación al $5^{\circ}$ día postoperatorio.

En la sala de fisioterapia, el día 39 de evolución presentó dos episodios de pérdida de conciencia con ausencia de pulso que recuperó tras escasos minutos de compresiones torácicas y ven-

Figura 1. A. Resonancia magnética nuclear: fractura del cuerpo vertebral de C6, anterolistesis grado IV de C6 sobre C7 con compresión medular y aplastamiento del borde anterosuperior del cuerpo vertebral de C7. B. Tomografía computarizada: artrodesis vertebral C6-C7 con autoinjerto más osteosíntesis.

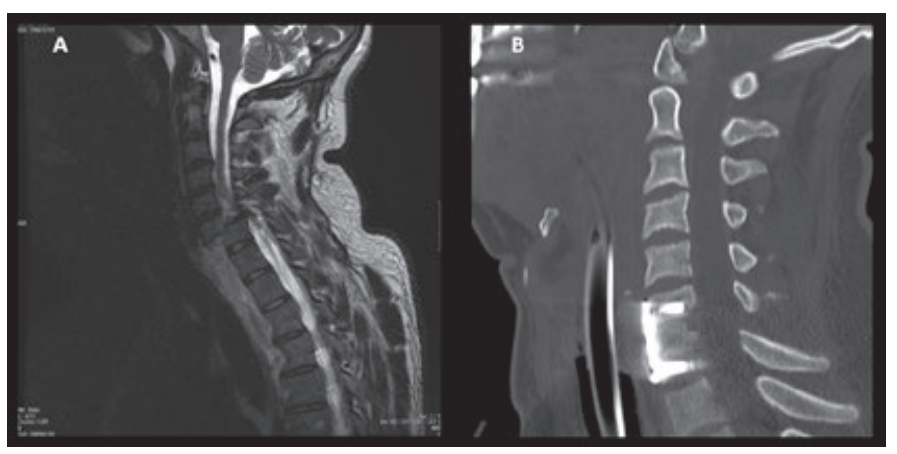


tilación con mascarilla y bolsa autoinflable conectada a oxígeno practicadas por el personal de la ULM. A la llegada del equipo de resucitación el paciente se encontraba hipotenso, con insuficiencia respiratoria y con una Escala de Coma de Glasgow de 15 puntos. A los pocos minutos sufrió una nueva PC aplicándose medidas de soporte vital avanzado (SVA) durante 45 minutos; dados los antecedentes y ante la evidencia de signos de TVP en la extremidad inferior izquierda con la sospecha de TEP masivo se administró trombolítico (alteplasa) intravenoso. Tras la RCE ingresó en la Unidad de Cuidados Intensivos 4 semanas. Se inició heparina sódica intravenosa con una evolución hemodinámica favorable; se confirmó TEP mediante angio-tomografía computarizada. Como complicaciones presentó infección urinaria bacteriémica por Klebsiella pneumoniae y dificultad para el destete del respirador que obligó a realizar una traqueostomía y solicitar un respirador portátil para el traslado nuevamente a la ULM. En las semanas sucesivas se retiró la ventilación mecánica, se realizó una gastrostomía endoscópica percutánea para nutrición y se mantuvo la anticoagulación terapéutica con enoxaparina $(1,5 \mathrm{mg} / \mathrm{kg} / 24 \mathrm{~h})$ por no alcanzar el efecto deseado con acenocumarol.

Se trasladó a un centro de Neurorrehabilitación para continuar el tratamiento neurorrehabilitador a las 22 semanas de ingreso hospitalario estable, con cánula de traqueostomía, episodios ocasionales de disautonomía vegetativa; neurológicamente su situación correspondía a un estado de vigilia con respuesta mínima.

\section{Discusión}

El riesgo de desarrollar una TVP surge de la presencia de la tríada de Virchow: hipercoagulabilidad, lesión del endotelio y estasis venosa; ${ }^{2-4}$ siendo la estasis, resultado de la inmovilización, uno de los factores más importantes en los lesionados medulares. ${ }^{2,4}$

La literatura expone una incidencia muy variable de TVP en la LMA, hasta 90 días después de la lesión. ${ }^{2-5}$ En los estudios es de un $10 \%$ a $100 \%$ sin profilaxis y de un $0 \%$ a un $7 \%$ con ella; ${ }^{3}$ demostrándose así que la profilaxis reduce de forma significativa la incidencia de TVP y su morbimortalidad. ${ }^{2-5}$ La evidencia sugiere que el empleo de las heparinas de bajo peso molecular (HBPM) es la medida más importante, ${ }^{2,4}$ sin que parezca existir diferencia entre los distintos tipos. ${ }^{2}$ La profilaxis con HBPM debe introducirse en las primeras 72 horas, ${ }^{2}$ y mantenerse hasta 3 meses, basándose en el reporte de un mayor riesgo de ETEV en ese periodo. ${ }^{2,4}$, ${ }^{5}$ Asimismo existe un beneficio intuitivo para la combinación con medidas físicas, ${ }^{2,4}$ aunque la evidencia a este respecto es limitada. ${ }^{4}$

Desde el punto de vista de la clasificación ASIA y del nivel neurológico, parece que el grado A y las lesiones cervicales bajas y torácicas altas respectivamente son los de mayor probabilidad de ETEV. ${ }^{2,5}$

La incidencia de TEP en la LMA oscila entre el 5-12\% con una tasa de mortalidad de hasta el 5\%, ${ }^{4,5}$ En el estudio de Nieto et al. el 53. 3\% de los pacientes con TVP presentaron un TEP. ${ }^{2}$ A pesar de que su incidencia disminuye una vez transcurridos los 3 primeros meses desde la lesión, ${ }^{5}$ argumento para suspender la profilaxis tras ese periodo, un estudio reciente muestra que el riesgo de TEP continúa siendo significativamente mayor en los lesionados medulares de más de 3 meses (incidencia del 1. 25\%) comparado con la población general (0.06\% por año). ${ }^{5}$

La piedra angular del tratamiento inicial de los TEP estables hemodinámicamente es la anticoagulación. Sin embargo, en el TEP masivo con inestabilidad hemodinámica o PC está indicada la reperfusión con trombolisis. ${ }^{1}$ El mecanismo primario por el que puede originar una PC es la oclusión del lecho arterial pulmonar por trombos que provoca una sobrecarga brusca de presión al ventrículo derecho (VD). Esto ocasiona una dilatación de un VD de paredes delgadas, alterando su capacidad contráctil, desviando el septo interventricular hacia la izquierda y como resultado impidiendo el llenado del ventrículo izquierdo que lleva a una reducción del gasto cardiaco que contribuye a la hipotensión sistémica, inestabilidad hemodinámica y PC. ${ }^{1}$ En una situación tan crítica, ante la imposibilidad de realizar las pruebas diagnósticas habituales (angiotomografía computarizada o gammagrafía pulmonar), una ecocardiografía a la cabecera del paciente que demuestre sobrecarga del VD justifica la reperfusión inmediata mediante trombolisis. ${ }^{1}$ Los mecanismos de acción por los que la trombolisis resulta beneficiosa en una PC por TEP masivo son: la liberación de la arteria pulmonar a través de la lisis total o parcial del trombo, la ayuda a la fragmentación de los trombos centrales compartida con la acción del masaje cardiaco externo, la fragmentación y lisis de los pequeños trombos pulmonares periféricos y la lisis de los trombos de la circulación venosa profunda que originan el cuadro limitando las recurrencias. ${ }^{8}$ Si se emplea la trombolisis en este contexto las guías clínicas consideran indicado prolongar la reanimación hasta 60-90 minutos antes de considerarla fallida y finalizarla. ${ }^{9}$

Las guías de reanimación cardiopulmonar (RCP) destacan que las principales medidas que contribuyen a mejorar la supervivencia de una PC son: iniciar un soporte vital básico efectivo con compresiones torácicas ininterrumpidas de alta calidad y la desfibrilación precoz en aquella situación en la que esté indicada (fibrilación ventricular y taquicardia ventricular sin pulso). ${ }^{9}$

Tras haber identificado la PC y solicitado ayuda hay que comenzar inmediatamente las maniobras de RCP manteniendo una relación compresión:ventilación 30:2 mientras el paciente no está intubado o con un dispositivo supraglótico de vía aérea (por ejemplo: mascarilla laríngea); debe mantenerse la vía aérea permeable y ventilar los pulmones con el equipamiento más adecuado inmediatamente disponible (habitualmente cánula orofaríngea y mascarilla con balón autoinflable conectado a una fuente de oxígeno); debe además analizarse el ritmo en cuanto se disponga de un desfibrilador externo automático (DESA) 0 un desfibrilador manual. $^{9}$

La lesión cerebral hipóxico-isquémica (LCHI) es una de las posibles consecuencias de una PC tras la RCE; su evolución y/o recuperación depende de la duración de la parada y la calidad de la reanimación. ${ }^{6}$ Ciertas áreas del cerebro son 
Tabla 1. Requisitos para el diagnóstico de estado vegetativo

Condiciones previas ineludibles
- Etiología conocida
- Situación no debida a efectos farmacológicos ni alteraciones
metabólicas
- Causas estructurales tratables descartadas por estudios de
neuroimagen
Criterios clínicos obligatorios
- Ausencia de evidencia de interrelación con el medio en ningún
momento
- Ausencia de respuesta a estímulos que indiquen voluntariedad o
propósito consciente
- Ausencia de evidencia de comprensión o emisión de lenguaje

Modificado de Alberdi et al.11

más vulnerables que otras, lo cual afecta al perfil temporal de recuperación: en primer lugar reaparecen las funciones primitivas del tronco encefálico (respiración y reflejos pupilares) seguido del retorno de la actividad en las estructuras profundas del cerebro y finalmente la recuperación de la actividad cortical y conciencia. ${ }^{6}$

Se considera un buen resultado neurológico después de una PC aquel paciente despierto con limitadas 0 ausentes secuelas neurológicas; ${ }^{6}$ la muerte 0 un deterioro grave que impida la vida independiente un mal resultado. ${ }^{10}$ Los profesionales de la salud debemos tener en cuenta que familiares y pacientes pueden tener diferentes percepciones de lo que constituye un resultado neurológico aceptable; ${ }^{10}$ en caso de plantearse la renuncia o retirada de medidas de soporte vital (si no existiese testamento vital) debería desarrollarse y discutirse con todas las personas involucradas en la situación (médico, familia) y con un apoyo multidisciplinar (psicólogos, especialistas en ética y expertos en comunicación). ${ }^{10}$ Así pues las secuelas derivadas de una LCHI secundaria a PC se sitúan entre dos polos: muerte y coma profundo en un extremo, y la recuperación funcional completa en el otro; entre ambos encontramos los estados vegetativos (EV) (tabla1) y los de mínima conciencia (EMC) (tabla2). ${ }^{11}$ Es posible confundir un EMC con un EV, especialmente en casos de grandes discapacidades motoras, ya que cualquier demostración de consciencia precisa una respuesta motriz. ${ }^{11}$

La PC por TEP es la presentación clínica más grave de la ETEV, en esta situación las guías de RCP consideran indicada la administración de un trombolítico intravenoso y continuar la RCP durante al menos 60-90 minutos antes de finalizarla. Esta recomendación se basa en la descripción de casos de recuperación de la circulación espontánea tras reanimaciones prolongadas después de administrar el trombolítico y con un buen resultado neurológico al alta. ${ }^{12} \mathrm{Sin}$ embargo, esas mismas guías destacan que la calidad de las compresiones torácicas en las PC intrahospitalarias es con frecuencia subóptima, lo cual condiciona un peor pronóstico para el paciente. En el caso que exponemos, a pesar de iniciarse una RCP precoz por personal sanitario entrenado y aplicarse la medidas de SVA durante 45 minutos hasta la RCE siguiendo las recomendaciones para la situación des-
Tabla 2. Criterios definitorios del estado de mínima conciencia

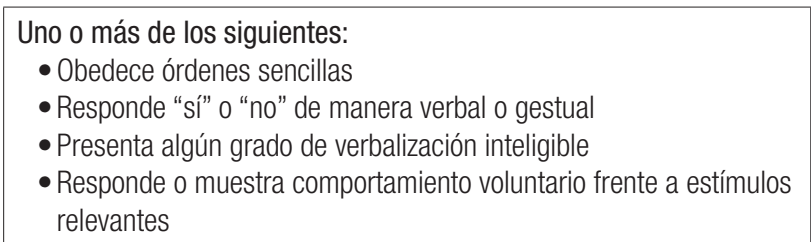

Modificado de Alberdi et al.11

crita, se obtuvo un mal resultado neurológico correspondiente a un EMC.

\section{Conclusiones}

- El TEP es una de las causas potencialmente reversibles de PC en un paciente con LMA.

- Todos los profesionales sanitarios deben saber reconocer una PC e iniciar la RCP con compresiones ininterrumpidas de alta calidad manteniendo la vía aérea permeable y ventilando los pulmones con el equipamiento disponible.

- La profilaxis con HBPM en la LMA reduce de forma significativa la TVP y su morbimortalidad.

- La LCHI es una de las posibles consecuencias de una PC recuperada.

\section{Bibliografía}

1. Konstantinides S, Torbicki A, Agnelli G, Danchin N, Fitzmaurice D, Galiè N et al. 2014 ESC Guidelines on the diagnosis and management of acute pulmonary embolism: the Task Force for the Diagnosis and Management of Acute Pulmonary Embolism of the European Society of Cardiology (ESC) Endorsed by the European Respiratory Society (ERS). Eur Heart J. 2014; 35: 3033-73.

2. Nieto-Blasco J, Martín-Mourelle R, Montoto-Marqués A, Ferreiro-Velasco ME, Salvador-de la Barrera S, Rodríguez-Sotillo A. Trombosis venosa profunda en la lesión medular traumática aguda. Rehabilitación. 2012; 46: 277-81.

3. Agarwal NK, Mathur N. Deep vein thrombosis in acute spinal cord injury. Spinal Cord. 2009; 47:769-72.

4. Teasell RW, Hsieh TJ, Aubut JL, Eng JJ, Krassioukov A, Tu L et al. Venous thromboembolism following spinal cord injury. Arch Phys Med Rehabil. 2009; 90: 232-45.

5. Alabed S, de Heredia LL, Naidoo A, Belci M, Hughes RJ, Meagher TM. Incidence of pulmonary embolism after the first 3 months of spinal cord injury. Spinal Cord. 2015; 53: 835-7.

6. Friberg H, Cronberg T. Prognostication after cardiac arrest. Best Pract Res Clin Anaesthesiol. 2013; 27: 359-72.

7. Bracken MB, Holford TR. Neurological and functional status 1 year after acute spinal cord injury: estimates of functional recovery in National Acute Spinal Cord Injury Study II from results modeled in National Acute Spinal Cord Injury Study III. J Neurosurg. 2002; 96(3 Suppl):259-66.

8. Ruiz-Bailén M, Rucabado-Aguilar L, Morante-Valle A, Castillo-Rivera A. Trombolisis en la parada cardíaca. Med Intensiva. 2006; 30: 62-7.

9. Monsieurs KG, Nolan JP, Bossaert LL, Greif R, Maconochie IK, Nikolaou Nl et al. European Resuscitation Council Guidelines for Resuscitation 2015: Section 1. Executive summary. Resuscitation. 2015; 95:1-80.

10. Geri G, Mongardon N, Daviaud F, Empana JP, Dumas F, Cariou A. Neurological consequences of cardiac arrest: where do we stand? Ann Fr Anesth Reanim. 2014; 33: 98-101.

11. Alberdi-Odriozola F, Iriarte-lbarrarán $M$, Mendía-Gorostidi $A$, Murgialdai $A$, Marco-Garde P. Pronóstico de las secuelas tras la lesión cerebral. Med Intensiva. 2009; 33: $171-81$.

12. Wu JP, Gu DY, Wang S, Zhang ZJ, Zhou JC, Zhang RF. Good neurological recovery after rescue thrombolysis of presumed pulmonary embolism despite prior 100 minutes CPR. J Thorac Dis. 2014; 6: E289-E293. 